\begin{tabular}{lc} 
Sharif University of Technology & Scientia Iranica \\
SCIENTIA & Transactions F: Nanotechnology \\
I RAN ICA & http://scientiairanica.sharif.edu \\
\hline
\end{tabular}

\title{
Prediction of the size of silver nanoparticles prepared via green synthesis: A gene expression programming approach
}

\author{
R. Sattari and G.R. Khayati* \\ Department of Materials Science and Engineering, Shahid Bahonar University of Kerman, Kerman, P.O. Box 76135-133, Kerman, \\ Iran.
}

Received 22 March 2019; received in revised form 7 February 2020; accepted 1 June 2020

\section{KEYWORDS}

Silver nanoparticle; Gene expression programming;

Particle size;

Green synthesis;

Sensitivity analysis.

\begin{abstract}
This study presents a new prediction model for estimating the size of silver nanoparticles (AgNPs) prepared by green synthesis via Gene Expression Programming (GEP). First, 30 different experiments were carried out to construct the GEP models. Plant extract, reaction temperature, concentration of silver nitrate $\left(\mathrm{AgNO}_{3}\right)$, and stirring time parameters were considered as input variables and the size of AgNPs was selected as the output variable. The collected experimental data were randomly divided into eight testing sets and 22 training sets for further analysis. By considering the correlation coefficient $\left(R^{2}\right)$, Mean Absolute Error (MAE), and Root Relative Square Error (RRSE) as the criteria, the performances of proposed models by GEP were compared. Finally, the best model (i.e., GEP-1) with $R^{2}=0.9961, \mathrm{MAE}=0.2545$, and RRSE $=0.0668$ was proposed as a new model with simplified mathematical expressions to estimate the size of AgNPs. The results of sensitivity analysis showed that the amount of plant extract, the concentration of $\mathrm{AgNO}_{3}$, stirring time, and reaction temperature were the most effective parameters on the size of AgNPs, respectively. The proposed model can be extended for a wide range of applications and it provides the possibility of minimum materials consumption in the preparation of the lowest-size AgNPs with regard to practical or economic constraints.
\end{abstract}

(C) 2020 Sharif University of Technology. All rights reserved.

\section{Introduction}

Silver nanoparticles (AgNPs) have desirable physical/chemical properties, including favorable optical and thermal characteristics as well as high electrical conductivity, and significant biological properties [1-

\footnotetext{
*. Corresponding author. Tel: +98-915-1903477; Fax: +98-341-2114053

E-mail addresses: roshan96sattari@gmail.com (R. Sattari); khayatireza@gmail.com; khayati@uk.ac.ir (G.R. Khayati)
}

doi: $10.24200 /$ sci. 2020.53209 .3112
3]. There are various chemical, physical, and biological methods for the synthesis of AgNPs. However, environmental hazards and high cost are the main drawbacks of the former two $[4,5]$. On the contrary, green synthesis has considerable advantages for the synthesis of AgNPs. Consequently, several researchers have tried to optimize the practical parameters of $\mathrm{Ag}$ NPs by green synthesis. However, unfortunately, this technique suffers from complicated interactions among the practical parameters as well as prolonged synthesis time [6]. According to the literature [7], particle size, composition, microstructure, and morphology of AgNPs significantly affect their unique features. As 
a consequence, employment of advanced optimization as well as modeling approaches to dictate the optimal properties of AgNPs is a hot topic for various studies. Accordingly, development of a detailed accurate model for the prediction of the size AgNPs will be so beneficial. The main merit of Gene Expression Programming (GEP) technique is its inherent capability to propose an equation as a mathematical function. This approach provides a systematic and efficient methodology to optimize the performance and quality of complicated engineering issues [8,9]. The main contribution of the current study is using GEP algorithm to estimate the particle size of $\mathrm{AgNPs}_{\mathrm{s}}$ with regard to experimental data, including plant extract $(P e)$, temperature of reaction $(T r)$, stirring time $(S t)$, and molar concentration of $\operatorname{AgNPs}_{(M c)}$, as effective practical parameters. A sensitivity analysis was performed to evaluate the effect of each input variable on the size of nanoparticles in the investigated range.

\section{Data collection and method of analysis}

\subsection{Data set and input/output selection}

Determining the important variables of green synthesis that affect the size of AgNPs is the main part of optimization and modeling. Shabanzadeh et al. [7] investigated the green synthesis of AgNPs using Vitax negundo L. extract by Artificial Neural Networks (ANNs). They mentioned that the AgNPs size was mainly affected by four practical parameters, namely $P e, T r, S t$, and $M c$. Table 1 summarizes the details of 30 practical data that repeated based on the operational condition of literature [7]. These data have randomly been divided into testing (eight cases) and training (22 cases) sets for the development of various GEP models.

\subsection{Gene Expression Programming (GEP)}

Genetic Programming (GP) is an enhanced version of Genetic Algorithm (GA) proposed by Koza [10]. Moreover, to overcome the limitations of both GA and GP algorithms, a new population-based evolutionary algorithm, named Gene Expression Programming (GEP), was introduced by Ferreira [11-13]. The inherent ability of GEP to develop an equation by considering independent practical parameters as input for estimating a predefined output with acceptable accuracy distinguishes it from other modeling approaches [14-17]. The main components of GEP are terminal set, termination condition, fitness function, control parameters, and function set [10]. Figure 1 illustrates the flowchart of a typical GEP. As observed, the main component of GEP consists in the genetic operators [18-20].

The operators of a gene function at level of chromosomes, hence promoting genetic diversity. It

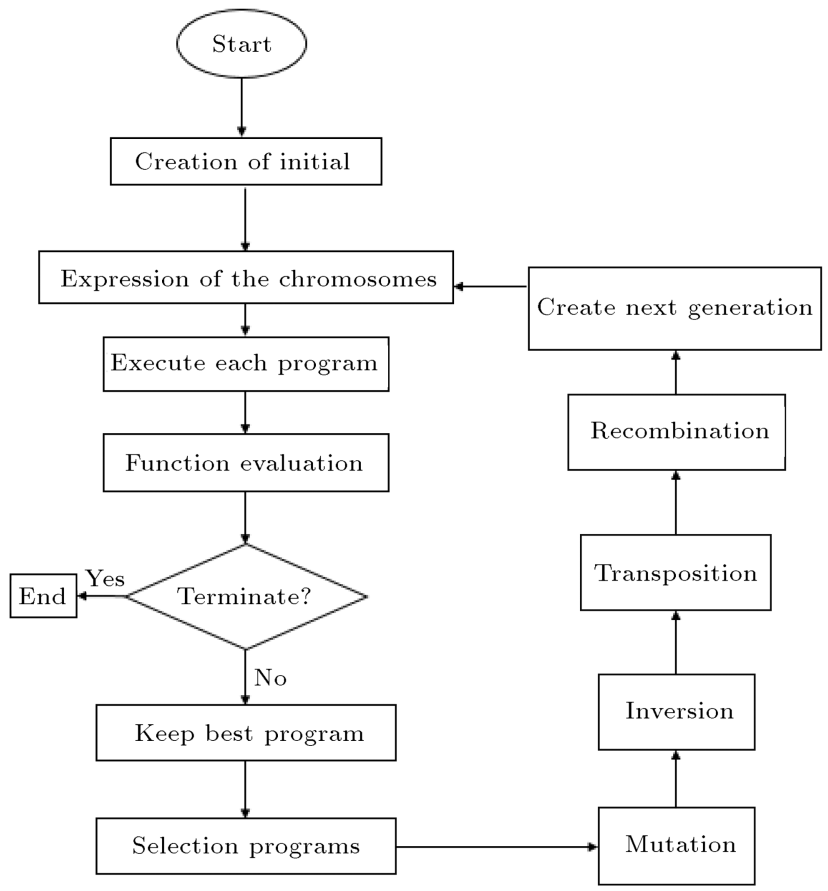

Figure 1. Flowchart of a GEP.

is noteworthy that GEP has a multi-gene nature and, consequently, each chromosome for one or more genes is a mathematical function. There are various methods to represent GEP output, including Karva language (the gene language), Expression Tree (ET), and mathematical functions [21-23]. Figure 2 shows a typical GEP output for an encoded chromosome with two genes as a linear string. Observing Figure 2 from left to right at the upper part shows the conversion of Karva language to ET. On the other hand, from right to left at the bottom, the figure illustrates the conversion of ET to a mathematical function [24].

To validate the randomly selected genome, the head-tail method was employed. In this methodology, every gene has two elements, i.e. a head and a tail. The former is composed of terminal and function, while the latter contains only the terminal symbols $[17,25]$. The GEP designer determines the head length $(h)$ and the length of the tail $(t)$ is calculated by Eq. (1) [26]:

$$
t=h\left(n_{\max }-1\right)+1,
$$

where $n_{\max }$ is the maximum number of arguments. GEP is performed in five major steps:

1. Selection of the fitness function $\left(f_{i}\right)$ through Eq. (2) [27]:

$$
f i=\sum_{j=1}^{C_{t}}\left(M-\left|C_{(i j)}-T_{j}\right|\right),
$$

where $M$ is the selection range, $C_{(i, j)}$ represents the return value of chromosome $i$ by employing the 
Table 1. Experimental data series for the synthesis of silver nanoparticles (AgNPs) by green synthesis [7] (plant extract $(P e)$, temperature of reaction $(T r)$, stirring time $(S t)$, and molar concentration of AgNPs $(M c))$.

\begin{tabular}{|c|c|c|c|c|c|}
\hline No. & $\begin{array}{c}P e^{*}(\mathrm{gr}) \\
\text { in } 100 \mathrm{ml} \text { water }\end{array}$ & $\begin{array}{l}\boldsymbol{T} \boldsymbol{r}^{* *} \\
\left({ }^{\circ} \mathrm{C}\right)\end{array}$ & $\begin{array}{c}S t^{* * *} \\
(\mathbf{h r})\end{array}$ & $\begin{array}{c}M c(\text { mole }) \\
\text { in } 100 \mathrm{~mL} \text { water }\end{array}$ & $\begin{array}{c}\text { AgNPs size } \\
(\mathrm{nm})\end{array}$ \\
\hline 1 & 0.10 & 25 & 48 & 0.1 & 27 \\
\hline 2 & 0.10 & 30 & 48 & 0.2 & 28 \\
\hline 3 & 0.10 & 40 & 48 & 0.5 & 29 \\
\hline 4 & 0.10 & 50 & 48 & 1.0 & 29 \\
\hline 5 & 0.10 & 60 & 48 & 1.5 & 31 \\
\hline 6 & 0.10 & 70 & 24 & 2.0 & 32 \\
\hline 7 & 0.25 & 25 & 24 & 0.1 & 25 \\
\hline 8 & 0.25 & 30 & 24 & 0.2 & 26 \\
\hline 9 & 0.25 & 40 & 24 & 0.5 & 26 \\
\hline 10 & 0.25 & 50 & 24 & 1.0 & 27 \\
\hline 11 & 0.25 & 60 & 12 & 1.5 & 27 \\
\hline 12 & 0.25 & 70 & 12 & 2.0 & 29 \\
\hline 13 & 0.50 & 25 & 12 & 0.1 & 18 \\
\hline 14 & 0.50 & 30 & 12 & 0.2 & 19 \\
\hline 15 & 0.50 & 40 & 12 & 0.5 & 21 \\
\hline 16 & 0.50 & 50 & 6 & 1.0 & 21 \\
\hline 17 & 0.50 & 60 & 6 & 1.5 & 24 \\
\hline 18 & 0.50 & 70 & 6 & 2.0 & 24 \\
\hline 19 & 0.75 & 25 & 6 & 0.1 & 15 \\
\hline 20 & 0.75 & 30 & 6 & 0.2 & 16 \\
\hline 21 & 0.75 & 40 & 3 & 0.5 & 18 \\
\hline 22 & 0.75 & 50 & 3 & 1.0 & 19 \\
\hline 23 & 0.75 & 60 & 3 & 1.5 & 20 \\
\hline 24 & 0.75 & 70 & 3 & 2.0 & 21 \\
\hline 25 & 1.00 & 25 & 3 & 0.1 & 16 \\
\hline 26 & 1.00 & 30 & 1 & 0.2 & 16 \\
\hline 27 & 1.00 & 40 & 1 & 0.5 & 17 \\
\hline 28 & 1.00 & 50 & 1 & 1.0 & 18 \\
\hline 29 & 1.00 & 60 & 1 & 1.5 & 18 \\
\hline 30 & 1.00 & 70 & 1 & 2.0 & 19 \\
\hline
\end{tabular}

fitness function, and $T_{j}$ stands for the target value corresponding to the fitness function $j$. If for all cases of $j$, the precision, i.e. $\left|C_{(i, j)}-T_{j}\right|$ is less than or equal to 0.01 , then $f_{i}=f_{\max }=C_{t} \times M$. In this study, $M$ is set to 100 and, consequently, $f_{\max }=1000$. In this way, the system is able to find the optimal solution on its own $[28,29]$;
2. Selection of (a) terminals $(S)$, (b) set of functions $(F)$ to generate the chromosomes as " $F "=(P e, T r, S t, M c)$, (c) arithmetic operators $(+,-, *, /)$, and (d) mathematical functions of Exp, pow10, Ln, Abs, $X^{2}, X^{3}, X^{4}, 3 R t$, Atan, and Cos;

3. Selection of the chromosomal architecture by de- 


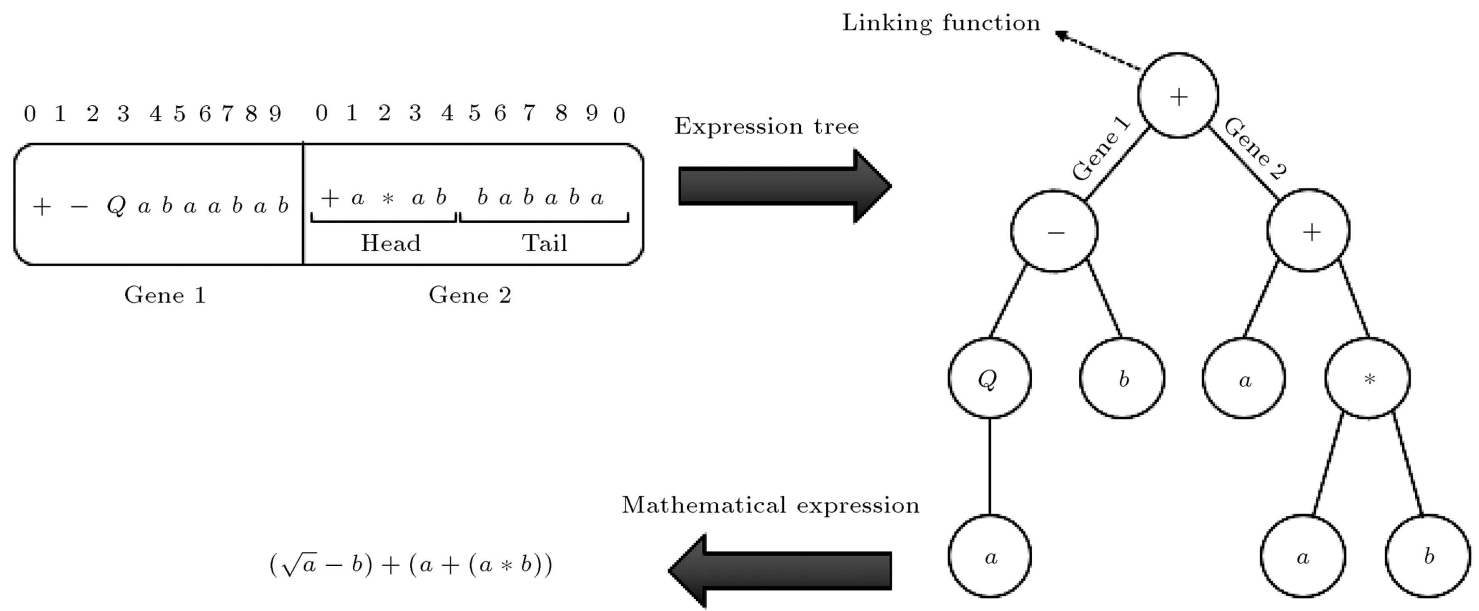

Figure 2. Representation of GEP output for a typical chromosome with two genes through mathematical expression, Expression Tree (ET), and Karva language.

Table 2. Predefined Gene Expression Progreamming (GEP) parameters for the most appropriate GEP models in the present study.

\begin{tabular}{lcccc}
\hline Model & Number of chromosomes & Head size & Number of genes & Used function \\
\hline GEP-1 & 24 & 10 & 6 & $+,-, *, /, \operatorname{Exp}, x^{2}, 3 R t$, Atan \\
GEP-2 & 28 & 10 & 5 & $+,-, *, /, \operatorname{Exp}_{,} x^{2}, x^{3}, x^{4}$ \\
GEP-3 & 36 & 10 & 6 & $+,-, *, /, \operatorname{Exp}, x^{2}, 3 R t$, Atan \\
GEP-4 & 30 & 9 & 4 & $+,-, *, /, \operatorname{Exp}$, Ln, Pow 10, Abs, $x^{4}$ \\
GEP-5 & 19 & 7 & 3 & $+,-, *, /, \operatorname{Exp}, x^{2}, 3 R t$ \\
GEP-6 & 25 & 4 & 3 &,$+ *$, Pow $10, x^{2}, 3 R t$, Cos \\
GEP-7 & 30 & 3 & 4 & $+,-, *, x^{2}, 3 R t$ \\
GEP-8 & 25 & 3 & 3 &,,$+- *$, Pow $10, x^{2}$, Cos \\
GEP-9 & 20 & 4 & 3 & $+,-, *, x^{2}, x^{3}$ \\
\hline
\end{tabular}

termining the number of genes and chromosomes and then, enhancing the length of heads one after another in every run. By considering performance as the criterion, the testing and training processes were monitored. Table 2 presents some trials for GEP modeling;

4. Selection of the linking function;

5. Determination of genetic operators as $[10,28]$ :

$\sqrt{ }$ Mutation [17,30]: The most efficient operator within the length of a chromosome with intrinsic modification power. Mutation is able to change the terminal or function in the head and the terminal in the tail;

$\sqrt{ }$ Inversion $[17,30]$ : Activated in the head of a chromosome. It is able to reverse a fragment with the length of 1 to 3 ;

$\sqrt{ }$ Transposition [17, 31]: Including three types of Insertion Sequence (IS) transposition, responsible for the transportation of a fragment or terminal from one position to the head or other genes; Root Insertion Sequence (RIS) transposition, responsible for transportation of a fragment by preserving its function from the first position to the changed root; and gene transposition, responsible for transporting the operators of all genes to the beginning of the chromosomes.

Table 3 shows the range of GEP parameters for the proposed models in this study. The powerful GeneXproTools 5.0 software was employed to model the relation between the practical parameters of green synthesis and the particle size of AgNPs. SPSS software (version 25) was employed to analyze the collected data as input variables. This study tried to predict the 
Table 3. Detailed features of Gene Expression Programming (GEP) for modeling.

\begin{tabular}{lc}
\hline Definition of GEP parameter & Value \\
\hline Number of chromosomes & $19-30$ \\
Head size & $3-10$ \\
Number of genes & $3-6$ \\
Linking function & Addition \\
Fitness function error type & RRSE \\
Constant per gene & 10 \\
Mutation rate & 0.0014 \\
Inversion rate & 0.5 \\
One-point recombination rate & 0.3 \\
Two-point recombination rate & 0.3 \\
Gene recombination rate & 0.3 \\
Gene transposition rate & 0.3 \\
IS transposition & 0.5 \\
RIS transposition & 0.5 \\
\hline
\end{tabular}

size of AgNPs prepared via green synthesis through the GEP approach. Hence, the practical parameters, i.e., $\mathrm{Pe}, \mathrm{Tr}, \mathrm{St}$, and $\mathrm{Mc}$, were selected as input and the size of AgNPs as output (Table 1). Among the 30 collected experimental data, 22 were randomly selected as the training set and the remaining eight were employed for testing to develop the GEP models of prediction.

\section{Result and discussion}

The presence of outlier practical data negatively affect clear understanding of the relationships between variables. Hence, identification and elimination of outlier data from the original data set leads to higher accuracy during GEP analysis. There are various techniques to detect the outliers, e.g., statistical and box plot approaches [32]. Figure 3 indicates the box plot for four
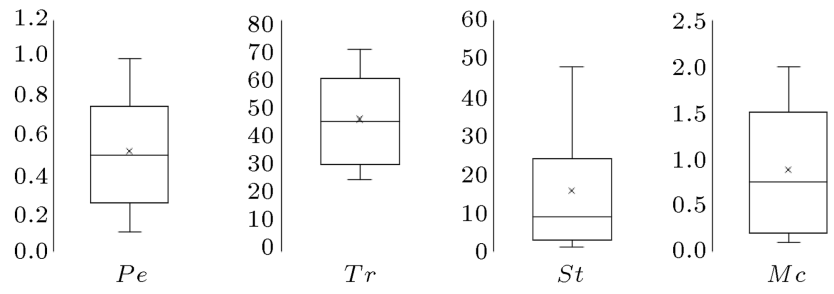

Figure 3. Boxplots of practical parameters for the green synthesis of AgNPs (plant extract $(P e)$, temperature of reaction $(T r)$, stirring time $(S t)$, and molar concentration of $\operatorname{AgNPs}(M c))$.

practical parameters during the preparation of AgNPs via green synthesis. The medians of the data sets for $P e$ and $T r$, which are situated in the center of the box, indicate their symmetric distribution. On the other hand, the box plots for $S t$ and $M c$ are inclined towards the bottom, that is, the boxes shift to the bottom whisker. This indicates that most of the values for the data are small with a few exceptions with large values. In these cases, the average is higher than the median and close to the upper whisker. It is noteworthy that all practical parameters in the present study do not have any outliers. Table 4 statistically presents the input and output data utilized in this study.

To determine the magnitude and direction of the relationships between practical parameters, Bivariate Correlation Analysis (BCA) was employed. BCA first performs a careful analysis of the way of measuring the practical data and then, determines any highly correlated pairs. Highly negative or positive correlation coefficients among the pairs decrease the accuracy of the developed GEP model, significantly, and complicate the evolving issues in explaining the effect of explanatory practical parameters on the size of AgNPs as the outcome. For example, in case of significant interdependency between the variables, the effect of each input parameter may be exaggerated, resulting in the evolution of multi collinearity [33]. Table 5 presents the correlation matrix of practical parameters by calculating of Pearson's coefficient. As indicated in the table, there are not any significant correlations between the independent variables of $\mathrm{Pe}, \mathrm{Tr}$, and $M c$. However, there is considerable dependency between the $M c$ and $T r$ as well as $S t$ and $P e$.

Table 4. Explanation of the statistical distribution of practical parameters in the development of Gene Expression Programming (GEP) models.

\begin{tabular}{cccccc}
\hline Parameter & Unit & Minimum & Maximum & Mean & Standard deviation \\
\hline $\boldsymbol{P e}$ & $\mathrm{gr}$ & 0.1 & 1 & 0.52 & 0.332 \\
$\boldsymbol{T r}$ & ${ }^{\circ} \mathrm{C}$ & 25 & 70 & 45.833 & 16.192 \\
$\boldsymbol{S t}$ & $\mathrm{hr}$ & 1 & 48 & 15.666 & 16.595 \\
$\boldsymbol{M} \boldsymbol{c}$ & $\mathrm{mL}$ & 0.1 & 2 & 0.883 & 0.703 \\
\hline
\end{tabular}


Table 5. Correlation coefficients of the practical variables for green synthesis of AgNPs; experimental data series for the synthesis of silver nanoparticles (AgNPs) by green synthesis [7] (plant extract $(P e)$, temperature of reaction $(T r)$, stirring time $(S t)$, and molar concentration of $\operatorname{AgNPs}(M c))$.

\begin{tabular}{ccccc}
\hline Parameters & $\boldsymbol{P e}$ & $\boldsymbol{T r}$ & $\boldsymbol{S t}$ & $\boldsymbol{M c}$ \\
\hline $\mathrm{Pe}$ & 1 & 0 & -0.845 & 0 \\
$\mathrm{Tr}$ & 0 & 1 & -0.186 & 0.993 \\
$\mathrm{St}$ & -0.845 & -0.186 & 1 & -0.191 \\
$\mathrm{Mc}$ & 0 & 0.993 & -0.191 & 1 \\
\hline
\end{tabular}

To study the multi collinearity between the practical parameters, a Principal Component Analysis (PCA) can be employed. PCA is an approach to dimension reduction in which the correlated variables are transferred from a multi-dimensional space to a lower-dimension space to remove the correlation of variables. The uncorrelated variables in the new space are called the principal component [34-36]. To ensure the possibility of performing PCA, the Kaiser Mayer Olkin (KMO) [37] factor should be adopted as the as criterion, calculated via Eq. (3).

$$
K M O=\frac{\sum_{i \neq j} \sum r i j^{2}}{\sum_{i \neq j} \sum r i j^{2}+\sum_{i \neq j} \sum a i j^{2}},
$$

where $r_{i j}$ and $a_{i j}$ are as the correlation coefficient and the practical correlation coefficient of variables $i$ and $j$, respectively. When the KMO factor is lower than 0.7 , the dependency between the practical parameters is unreal and the data are not appropriate for PCA analysis [32]. The KMO factor the in current study was estimated at 0.502 . Therefore, there was no need to use PCA for the correction of the interaction between the practical parameters. The KMO analysis revealed that the presence of considerable dependency between some of the practical parameters in Table 4 was a consequence of the nature of the BCA analysis, not a causality or linear association of $M c$ with $T r$ or $S t$ with $P e$. Thus, the practical parameters are independent from each other and appropriate for the subsequent analysis by GEP.

Validation of every developed model was performed by considering the coefficient of determination $\left(R^{2}\right)$, Mean Absolute Error (MAE), and Root Relative Square Error (RRSE) as the criteria, defined through Eqs. (4)-(6) [38].

$$
\begin{aligned}
& R^{2}=\frac{\left(n \sum t_{i} o_{i}-\sum t_{i} \sum o_{i}\right)^{2}}{\left(n \sum t_{i}{ }^{2}-\left(\sum t_{i}\right)^{2}\right)\left(n \sum o_{i}{ }^{2}-\left(\sum o_{i}\right)^{2}\right.}, \\
& R R S E=\sqrt{\frac{\sum_{i=1}^{n}(t i-o i)^{2}}{\sum_{i=1}^{n}\left(t i-\frac{1}{n} \sum_{i=1}^{n} t i\right)^{2}},} \\
& M A E=\frac{\sum_{i=1}^{n}|t i-o i|}{n},
\end{aligned}
$$

where $t$ is the target value, $o$ is the predicted value, and $n$ is the number of data within the testing and training phases. $R^{2}$ closer to 1 and MAE and RRSE closer to zero indicate better fit of the developed model [39]. The statistical characteristics of nine appropriate models for the training and testing data sets are given in Table 6 .

As indicated, the values of $R^{2}$ for the proposed

\begin{tabular}{|c|c|c|c|c|c|c|}
\hline \multirow[b]{3}{*}{ No. } & \multirow{2}{*}{\multicolumn{2}{|c|}{$R^{2}$}} & \multicolumn{4}{|c|}{ Error } \\
\hline & & & \multicolumn{2}{|c|}{ Training } & \multicolumn{2}{|c|}{ Testing } \\
\hline & Training & Testing & MAE & RRSE & MAE & RRSE \\
\hline GEP-1 & 0.9983 & 0.9961 & 0.1403 & 0.041 & 0.2545 & 0.0668 \\
\hline GEP-2 & 0.9902 & 0.9798 & 0.4055 & 0.0988 & 0.6428 & 0.1616 \\
\hline GEP-3 & 0.9954 & 0.9963 & 0.2586 & 0.0683 & 0.3193 & 0.0951 \\
\hline GEP-4 & 0.9905 & 0.9930 & 0.3834 & 0.0971 & 0.4185 & 0.1026 \\
\hline GEP-5 & 0.9857 & 0.9895 & 0.5101 & 0.1195 & 0.4141 & 0.1129 \\
\hline GEP-6 & 0.9854 & 0.9915 & 0.4795 & 0.1206 & 0.4877 & 0.1274 \\
\hline GEP-7 & 0.9826 & 0.9906 & 0.5220 & 0.1316 & 0.5018 & 0.1297 \\
\hline GEP-8 & 0.9855 & 0.9865 & 0.4724 & 0.1200 & 0.5310 & 0.1450 \\
\hline GEP-9 & 0.9827 & 0.9884 & 0.5153 & 0.1316 & 0.5138 & 0.1342 \\
\hline
\end{tabular}
model range between $0.9826-0.9983$ and $0.9798-0.9963$

Table 6. Correlation coefficient $\left(R^{2}\right)$, Mean Absolute Error (MAE), and Root Relative Square Error (RRSE) amounts for nine appropriate GEP models. 


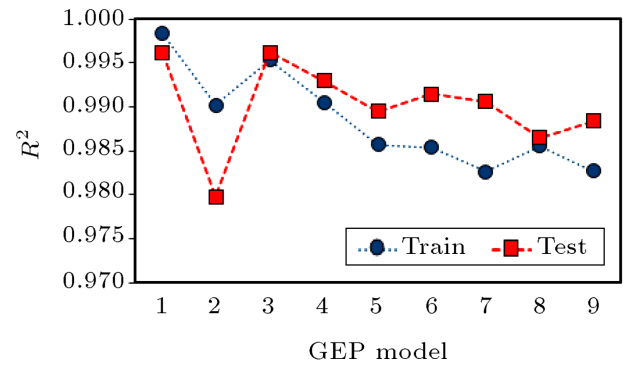

(a)

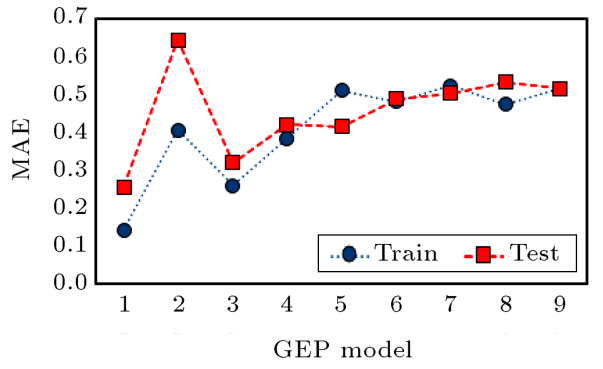

(b)

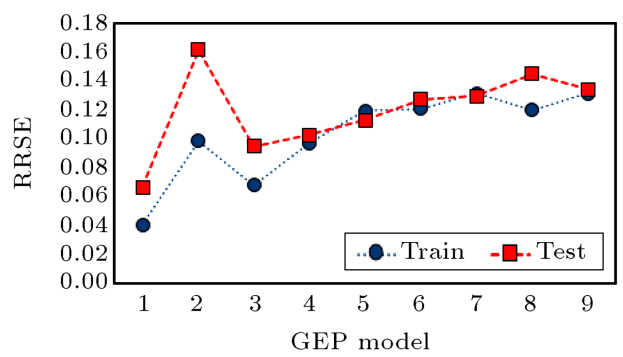

(c)

Figure 4. Various statistical indices for training and testing data series in Gene Expression Programming (GEP) models: (a) Correlation coefficient $\left(R^{2}\right)$, (b) Mean Absolute Error (MAE), and (c) Root Relative Square Error (RRSE).
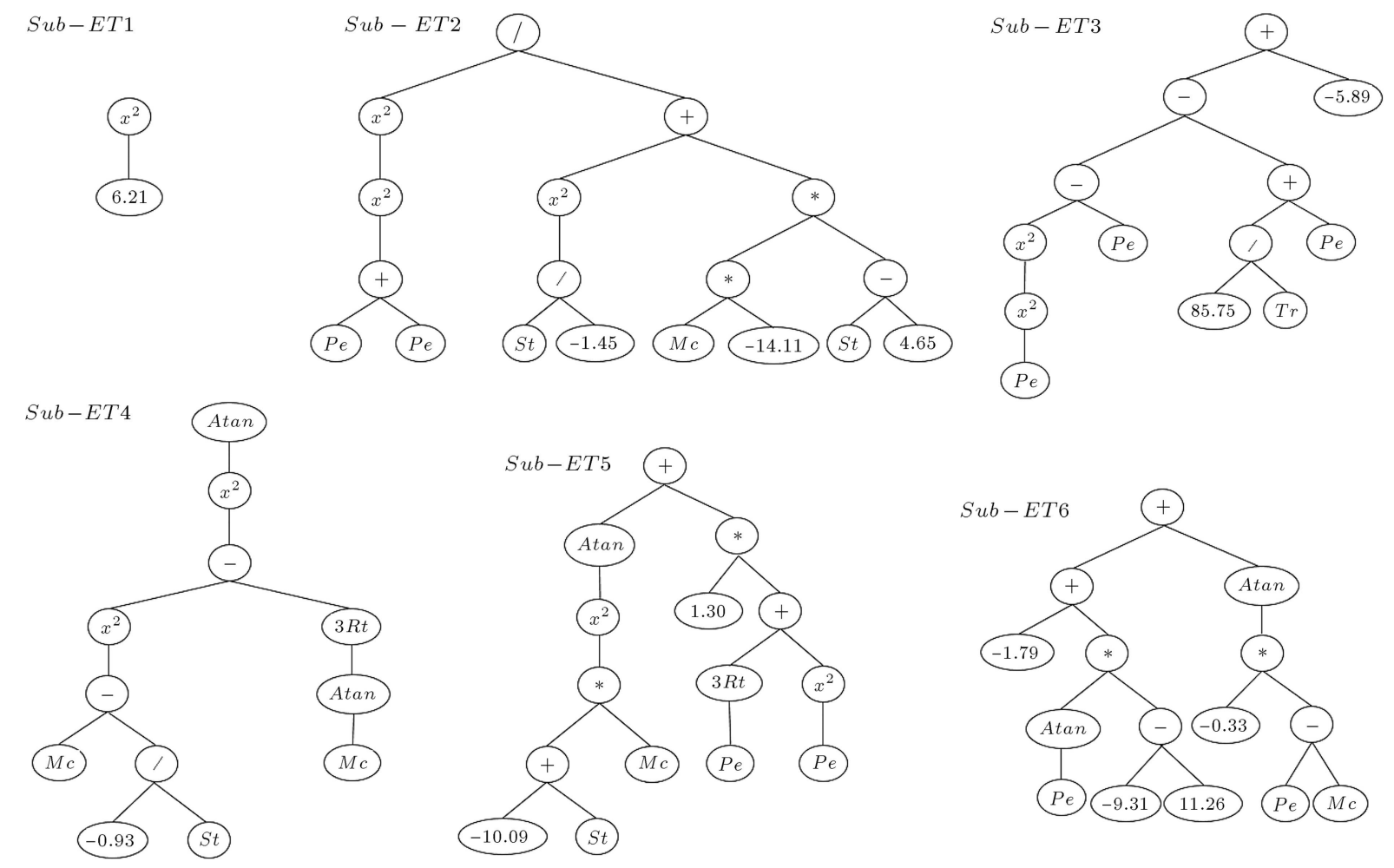

Figure 5. Expression Tree (ET) of the GEP-1 model.

in the training and testing phases, respectively. Moreover, the minimum amounts of MAE and RRSE are equal to 0.1403 and 0.041 in the training phase and 0.2545 and 0.0668 in the testing phase, respectively. Figure 4 compares the values of $R^{2}$, MAE, and RRSE for nine better GEP models. GEP-1, GEP-3, and GEP-
4 show higher accuracies than other models for the prediction of AgNPs size.

Table 7 summarizes the mentioned nine better GEP models. The relatively large size of GEP models shows the complicated space for the practical parameters of green synthesis. Figures 5-7 illustrated 
Table 7. The equations extracted from the nine Gene Expression Programming (GEP) models with experimental data series for the synthesis of silver nanoparticles (AgNPs) through green synthesis [7] (plant extract $(P e)$, temperature of reaction $(\mathrm{Tr})$, stirring time $(S t)$, and molar concentration of $\mathrm{AgNPs}(M c)$ ).

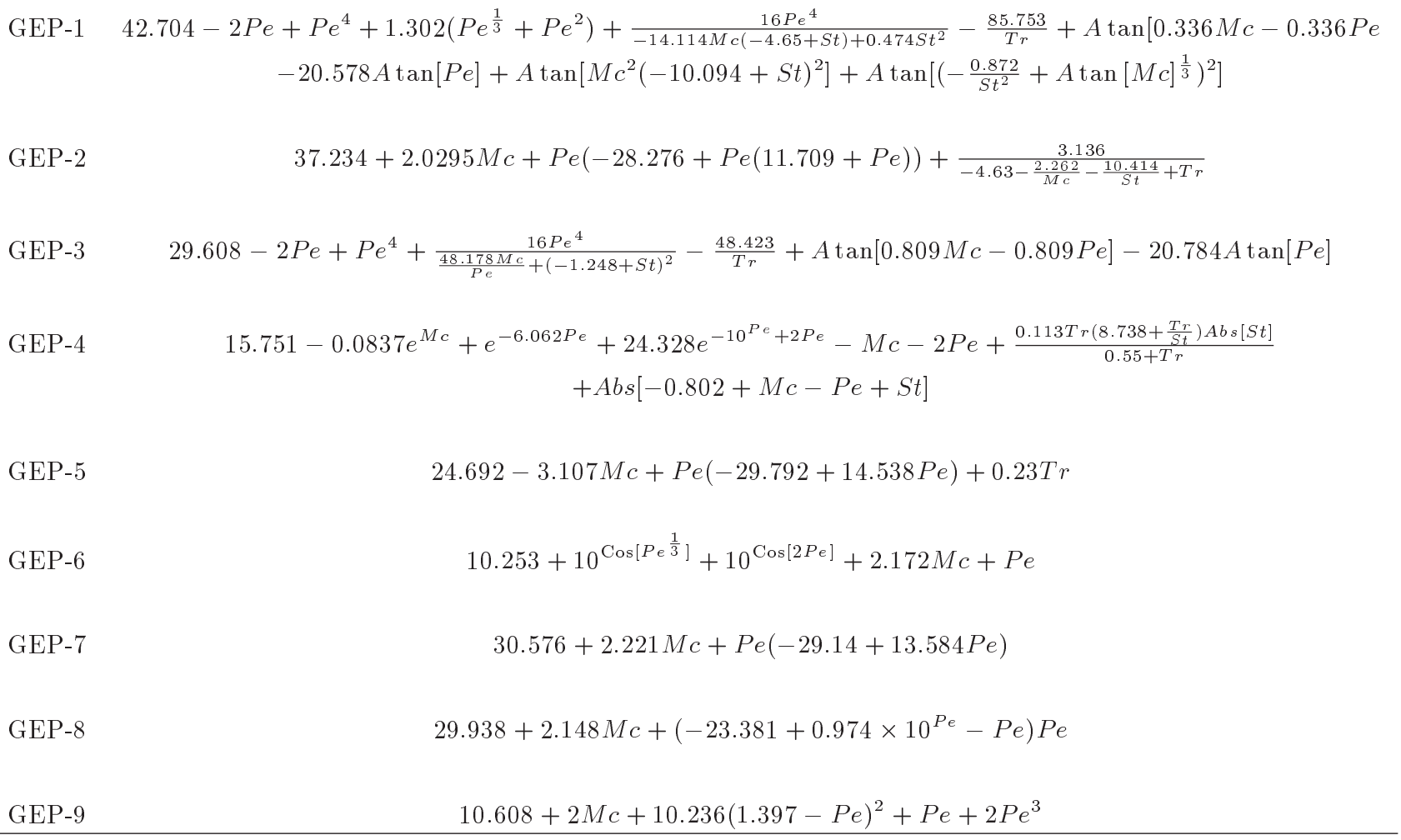

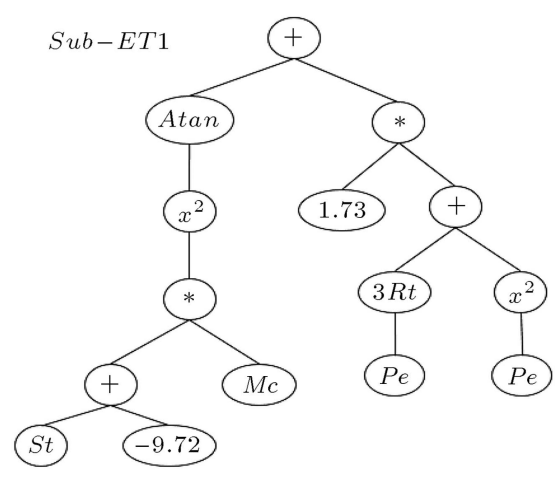
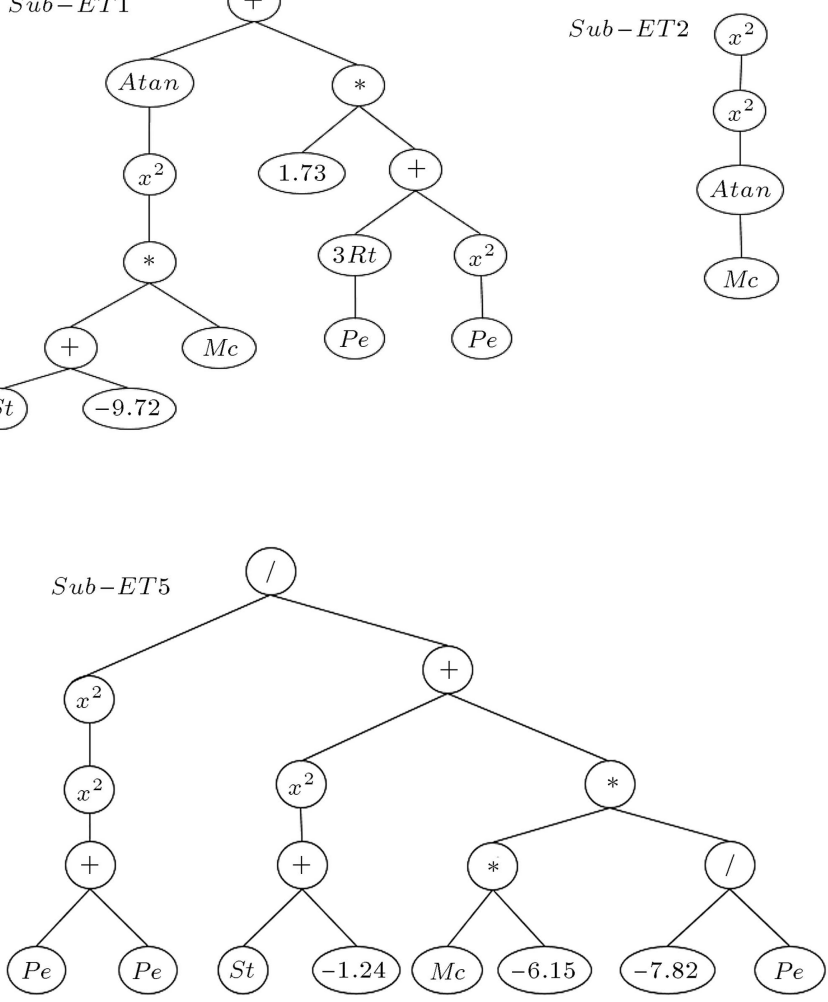

Figure 6. Expression Tree (ET) of the GEP-3 model.
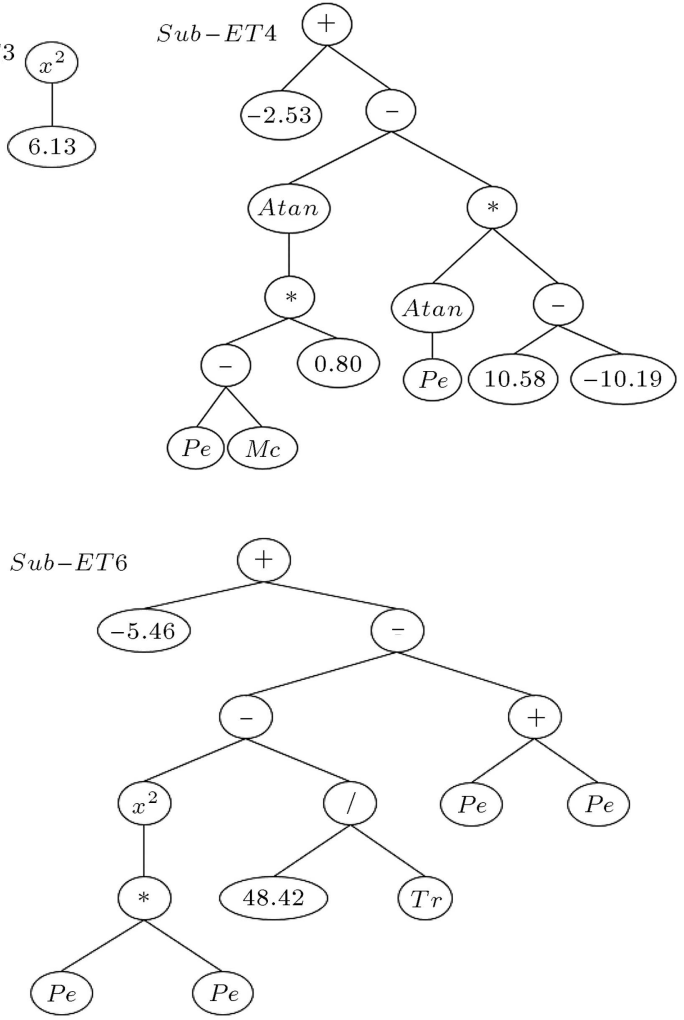

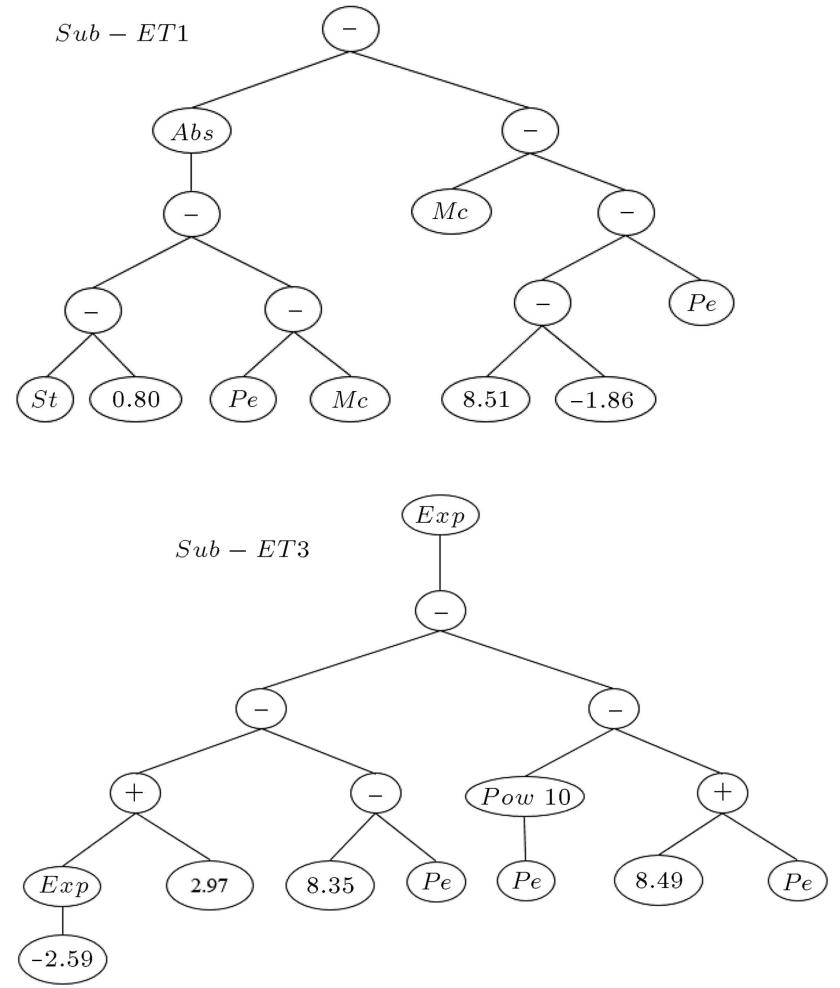
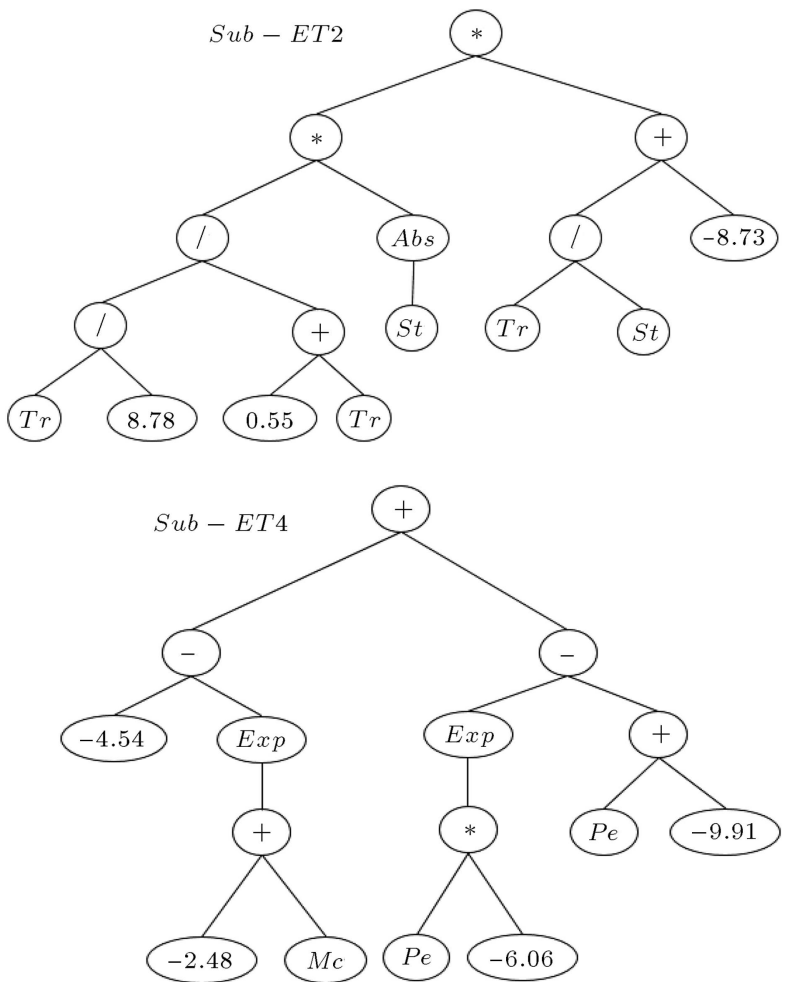

Figure 7. ET of the GEP-4 model.

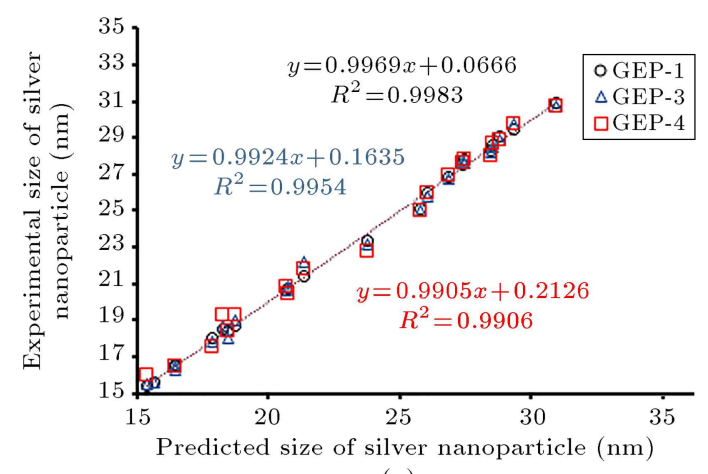

(a)

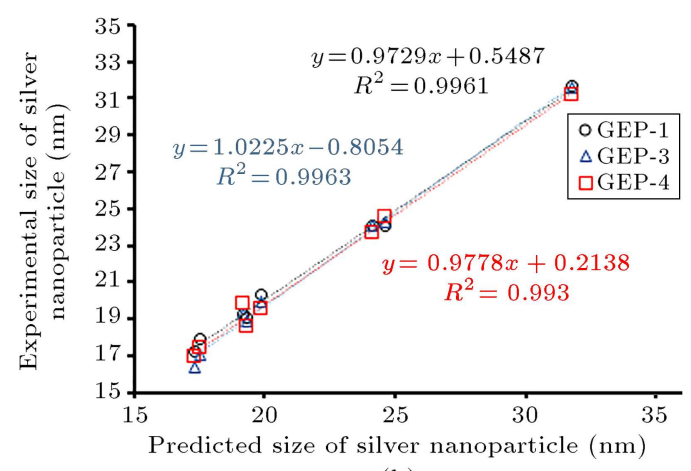

(b)

Figure 8. Comparison of actual and predicted values of AgNPs size by GEP-1, GEP-3, and GEP-4 for (a) the training phase and (b) the testing phase.

the sub-ETs of GEP-1, GEP-3, and GEP-4 models, respectively. The number of genes $(s u b-E T s)$ is 6 for GEP-1, 8 for GEP-2, 6 for GEP-3, and 4 for GEP-4 models. Suppose $m$ is the maximum number of iterations, $n$ is the number of chromosomes, and $O(e)$ indicates the complexity of each GEP model. Then, time complexity of our strategy is $O(m n e)$, since in each iteration, the GEP model is executed for each chromosome.

Table 8 compares the actual and predicted sizes of AgNPs for GEP-1, GEP-3, and GEP-4 models. The results confirm that the proposed models are unable to predict the size of AgNPs with an acceptable precision.
Figure 8 illustrates the actual and predicted values of AgNPs size for GEP-1, GEP-3, and GEP4 in the testing and training phases. As shown, there is reasonable agreement between the experimental and predicted values for the GEP-1, GEP-3, and GEP-4, especially in the training phase. However, some deviations are observed from the linear trend, especially in GEP-3 and GEP-4 during the testing phase. Accordingly, GEP-1 is proposed as the most appropriate model for the estimation of the size of AgNPs prepared by green synthesis.

A sensitivity analysis is performed by changing an output/input and keeping all other parameters constant [40]. To estimate the relative influence of every 
Table 8. Comparison of the actual and predicted AgNPs sizes with GEP-1, GEP-3, and GEP-4 models.

\begin{tabular}{|c|c|c|c|c|}
\hline \multirow[t]{2}{*}{ No. } & \multirow[t]{2}{*}{ Actual AgNPs size (nm) } & \multicolumn{3}{|c|}{ Predicted AgNPs (nm) } \\
\hline & & GEP-1 & GEP-3 & GEP-4 \\
\hline 1 & 27.39 & 27.54 & 27.73 & 27.59 \\
\hline 2 & 28.44 & 28.27 & 28.18 & 27.98 \\
\hline 3 & 28.83 & 29.07 & 28.88 & 28.87 \\
\hline 4 & 29.31 & 29.43 & 29.77 & 29.79 \\
\hline 5 & 30.98 & 30.90 & 30.71 & 30.70 \\
\hline 6 & 31.79 & 31.65 & 31.54 & 31.23 \\
\hline 7 & 24.62 & 24.07 & 24.28 & 24.52 \\
\hline 8 & 25.77 & 25.10 & 25.02 & 24.99 \\
\hline 9 & 26.08 & 25.99 & 25.81 & 25.99 \\
\hline 10 & 26.84 & 26.83 & 26.75 & 26.98 \\
\hline 11 & 27.49 & 27.83 & 27.63 & 27.76 \\
\hline 12 & 28.53 & 28.55 & 28.51 & 28.64 \\
\hline 13 & 18.23 & 18.46 & 18.65 & 19.30 \\
\hline 14 & 19.21 & 19.24 & 19.20 & 19.82 \\
\hline 15 & 20.67 & 20.63 & 20.60 & 20.87 \\
\hline 16 & 21.32 & 21.40 & 22.15 & 21.79 \\
\hline 17 & 23.78 & 23.39 & 23.20 & 22.77 \\
\hline 18 & 24.12 & 24.03 & 24.09 & 23.66 \\
\hline 19 & 15.37 & 5.40 & 15.49 & 15.95 \\
\hline 20 & 16.43 & 16.53 & 16.22 & 16.49 \\
\hline 21 & 17.83 & 17.99 & 17.86 & 17.51 \\
\hline 22 & 19.33 & 19.06 & 18.83 & 18.55 \\
\hline 23 & 19.85 & 20.28 & 19.88 & 19.54 \\
\hline 24 & 20.74 & 20.75 & 20.80 & 20.43 \\
\hline 25 & 15.64 & 15.60 & 15.63 & 14.73 \\
\hline 26 & 16.44 & 16.44 & 16.46 & 16.44 \\
\hline 27 & 17.31 & 17.19 & 16.38 & 16.93 \\
\hline 28 & 17.55 & 7.94 & 17.05 & 17.38 \\
\hline 29 & 18.47 & 18.38 & 18.04 & 18.36 \\
\hline 30 & 18.72 & 18.72 & 18.97 & 19.26 \\
\hline
\end{tabular}

practical parameter on the AgNPs size, sensitivity analysis of the most appropriate model, i.e., GEP-1, was carried out (Figure 9). As shown, plant extract, concentration of $\mathrm{AgNO}_{3}$, and stirring time had positive effects on reducing the AgNPs size with plant extract being the most effective parameter. Furthermore, reaction temperature, with negligibly negative effect within the investigated range, had the minimum effect on the size of AgNPs.

Figure 10 illustrates the effects of two most influential parameters $(M c$ and $P e$ ) on the AgNPs size in 3D profiles. As shown, decreasing Pe from 1 


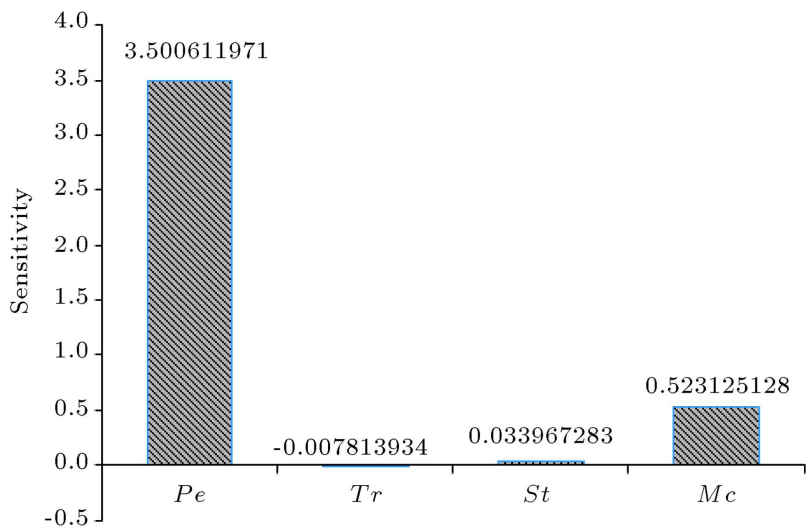

Figure 9. Sensitivity analysis of the practical parameters for the green synthesis of AgNPs (plant extract $(\mathrm{Pe})$, temperature of reaction $(T r)$, stirring time $(S t)$, and molar concentration of $\operatorname{AgNPs}(M c))$.

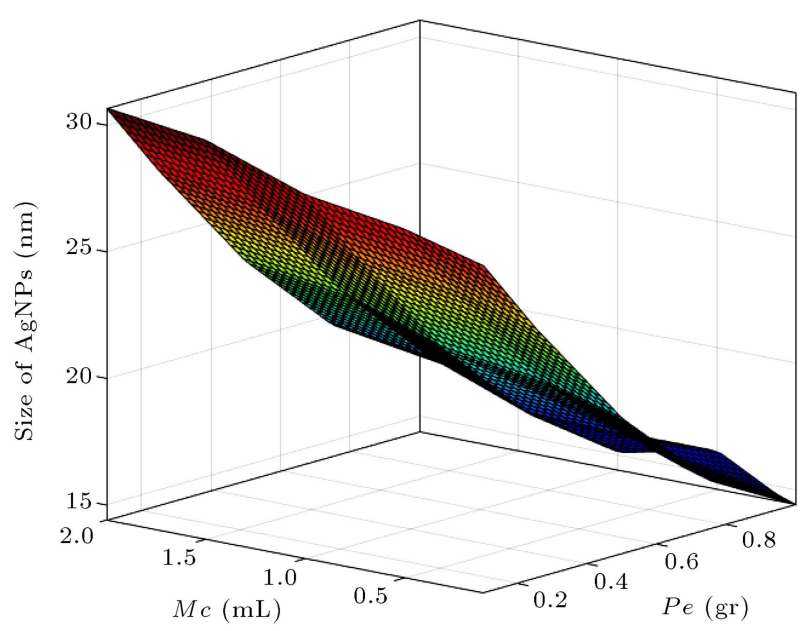

Figure 10. 3D profiles of AgNPs changes with changes in the most important parameters (plant extract $(P e)$ and molar concentration of AgNPs $(M c)$ ) for green synthesis when other practical parameters $(\operatorname{Tr}$ (temperature of reaction $)=40^{\circ} \mathrm{C}$ and $S t$ (stirring time $)=12 \mathrm{hr}$ ) are constant.

to 0 caused to a severer enhancement in AgNPs size, even at higher levels of $M c$ (e.g. 1.5). That is, $P e$ is the major parameter in the synthesis and any increase in $P e$ leads to a significant decrease in the AgNPs size.

\section{Conclusion}

AgNPs size is a crucial physical/chemical property that significantly affects the quality of products. Unique characteristics of green synthesis as an appropriate alternative to the conventional methods encouraged us to estimate AgNPs size prepared through green synthesis. In summary, this study tried to employ Gene Expression Programming (GEP) for the estimation of AgNPs size on the basis of experimentally collected data. Various functions and architectures of modeling under different preparation conditions were compared to find the most appropriate GEP model. The results showed that GEP-1 had the best performance in the prediction of particle size with RRSE, $R^{2}$, and Mean Absolute Error (MAE) equal to 0.0668, 0.9961, and 0.2545 , respectively. Also, sensitivity analysis of GEP1 revealed that plant extract and concentration of $\mathrm{AgNO}_{3}$ were the most effective parameters on the size of AgNPs.

\section{Nomenclature}

$\begin{array}{ll}\text { GEP } & \text { Gene Expression Programming } \\ P e & \text { Plant extract } \\ T r & \text { Temperature of reaction } \\ S t & \text { Stirring time } \\ M c & \text { Molar concentration of AgNPs } \\ R^{2} & \text { Correlation coefficient } \\ \text { MAE } & \text { Mean Absolute Error } \\ \text { RRSE } & \text { Root Relative Square Error } \\ \text { ET } & \text { Expression Tree } \\ h & \text { Head of gene } \\ t & \text { Tail of gene } \\ \text { RIS } & \text { Root Insertion Sequence transposition } \\ \text { IS } & \text { Insertion Sequence } \\ \text { BCA } & \text { Bivariate Correlation Analysis } \\ \text { PCA } & \text { Principal Component Analysis } \\ \text { KMO } & \text { Kaiser Mayer Olkin }\end{array}$

\section{References}

1. Santra, T.S., Tseng, F.-G.K., and Barik, T.K. "Biosynthesis of silver and gold nanoparticles for potential biomedical applications-A brief review", $J$ Nanopharm Drug Deliv, 2(4), pp. 249-265 (2014).

2. Saber, M.M., Mirtajani, S.B., and Karimzadeh, K. "Green synthesis of silver nanoparticles using Trapa natans extract and their anticancer activity against A431 human skin cancer cells", J Drug Deliv Sci Tech, 47, pp. 375-379 (2018).

3. Pirtarighat, S., Ghannadnia, M., and Baghshahi, S. "Antimicrobial effects of green synthesized silver nanoparticles using Melissa officinal is grown under in vitro condition", Nanomed J, 4(3), pp. 184-190 (2017).

4. Govindaraju, K., Basha, S.K., Kumar, V.G., et al. "Silver, gold and bimetallic nanoparticles production using single-cell protein (Spirulina platensis) Geitler", J. Mater. Sci, 43(15), pp. 5115-5122 (2008).

5. Kumar, A., Mandal, S., Selvakannan, P., et al. "Investigation into the interaction between surfacebound alkylamines and gold nanoparticles", Langmuir, 19(15), pp. 6277-6282 (2003). 
6. Mukherjee, S., Nethi, S.K., and Patra, C.R. "Green synthesized gold nanoparticles for future biomedical applications", Particulate Technology for Delivery of Therapeutics, Springer, pp. 359-393 (2017).

7. Shabanzadeh, P., Yusof, R., and Shameli, K. "Artificial neural network for modeling the size of silver nanoparticles' prepared in montmorillonite/starch bionanocomposites", J Ind Eng Chem, 24, pp. 42-50 (2015).

8. Behnia, D., Ahangari, K., Goshtasbi, K., et al. "Settlement modeling in central core rockfill dams by new approaches", Int. J. Min. Sci. Technol, 26(4), pp. 703$710(2016)$.

9. Jafari, M.M. and Khayati, G.R. "Prediction of hydroxyapatite crystallite size prepared by sol-gel route: gene expression programming approach", J Sol-Gel Sci Techn, 86(1), pp. 112-125 (2018).

10. Koza, J.R., Genetic Programming II, Automatic Discovery of Reusable Subprograms: MIT Press, Cambridge, MA (1992).

11. Kayadelen, C. "Soil liquefaction modeling by genetic expression programming and neuro-fuzzy", Expert Syst Appl, 38(4), pp. 4080-4087 (2011).

12. Khademalrasoul, A. and Adib, A. "Estimation of mixed-mode fracture parameters by gene expression", Sci Iran, 27(1), pp. 229-238 (2018).

13. Iqbal, M.F., Liu, Q.-F., Azim, I., et al. "Prediction of mechanical properties of green concrete incorporating waste foundry sand based on gene expression programming", J. hazard. Mater, 384, pp. 1-17 (2020).

14. Ferreira, C., Gene Expression Programming: Mathematical Modeling by an Artificial Intelligence, Springer (2006).

15. Teodorescu, L. and Sherwood, D. "High energy physics event selection with gene expression programming", Comput Phys Commun, 178(6), pp. 409-419 (2008).

16. Khandelwal, M., Faradonbeh, R.S., Monjezi, M., et al. "Function development for appraising brittleness of intact rocks using genetic programming and non-linear multiple regression models", Eng Comput-Germany, 33(1), pp. 13-21 (2017).

17. Hoseinian, F.S., Faradonbeh, R.S., Abdollahzadeh, A., et al. "Semi-autogenous mill power model development using gene expression programming", Powder Technol, 308, pp. 61-69 (2017).

18. Ferreira, C. "Gene expression programming: A new adaptive algorithm for solving problems", Complex Syst, 13(2), pp. 87-129 (2001).

19. Sarıdemir, M. "Effect of specimen size and shape on compressive strength of concrete containing fly ash: Application of genetic programming for design", Mater Design (1980-2015), 56, pp. 297-304 (2014).
20. Yekani Motlagh, S., Sharifi, A., Ahmadi, M., et al. "Presentation of new thermal conductivity expression for $\mathrm{Al} 2 \mathrm{O} 3$-water and $\mathrm{CuO}$-water nanofluids using gene expression programming (GEP)", J Therm Anal Calorim, 135(1), pp. 195-206 (2019).

21. Abdellahi, M., Bhmanpour, M., and Bahmanpour, M. "Optimization of process parameters to maximize hardness of metal/ceramic nanocomposites produced by high energy ball milling", Ceram Int, 40(10), pp. 16259-16272 (2014).

22. Keshavarz, A. and Tofighi, H. "Gene expression programming models for liquefaction-induced lateral spreading", Sci Iran, In press (2018). Gene expression programming models for liquefaction-induced lateral spreading (doi.org)

23. Bhowmik, S., Paul, A., Panua, R., et al. "Artificial intelligence based gene expression programming (GEP) model prediction of Diesel engine performances and exhaust emissions under Diesosenol fuel strategies", Fuel, 235, pp. 317-325 (2019).

24. Abdellahi, M., Bahmanpour, M., and Bahmanpour, M. "Laminating; the best way to improve Charpy impact energy of nanocomposites", Ceram Intl, 40(10), pp. 16115-16125 (2014).

25. Hosseini, S.S.S. and Gandomi, A.H. "Short-term load forecasting of power systems by gene expression programming", Neural Comput Appl, 21(2), pp. 377-389 (2012).

26. Liu, L.-W. and Wang, Y.-M. "Modelling reservoir turbidity using landsat 8 satellite imagery by gene expression programming", Water, 11(7), p. 1479 (2019).

27. Abdellahi, M., Karafshani, M.K., and Rizi, A.S. "Modeling effect of SiO2 nanoparticles on the mechanical properties of the concretes", Journal of Building Pathology and Rehabilitation, 2(1), p. 8 (2017).

28. Ferreira, C. Gene expression programming in problem solving. Soft computing and industry: Springer, pp. 635-653 (2002).

29. Aytek, A. and Alp, M. "An application of artificial intelligence for rainfall-runoff modeling", J Earth Syst Sci, 117(2), pp. 145-155 (2008).

30. Baykasoğlu, A., Güllü, H., Çanakçı, H., et al. "Prediction of compressive and tensile strength of limestone via genetic programming", Expert Syst Appl, 35(1-2), pp. 111-123 (2008).

31. Armaghani, D.J., Safari, V., Fahimifar, A., et al. "Uniaxial compressive strength prediction through a new technique based on gene expression programming", Neural Comput Appl, 30(11), pp. 3523-3532 (2018).

32. Faradonbeh, R.S. and Monjezi, M. "Prediction and minimization of blast-induced ground vibration using two robust meta-heuristic algorithms", Eng ComputGermany, 33(4), pp. 835-851 (2017).

33. Mousavi, S.M., Aminian, P., Gandomi, A.H., et al. "A new predictive model for compressive strength of HPC using gene expression programming", Adv Eng Softw, 45(1), pp. 105-114 (2012). 
34. Tiryaki, B. "Predicting intact rock strength for mechanical excavation using multivariate statistics, artificial neural networks, and regression trees", Eng Geology, 99(1-2), pp. 51-60 (2008).

35. Sayadi, A.R., Khalesi, M.R., and Borji, M.K. "A parametric cost model for mineral grinding mills", Miner Eng, 55, pp. 96-102 (2014).

36. Sayadi, A.R., Lashgari, A., and Paraszczak, J.J. "Hard-rock LHD cost estimation using single and multiple regressions based on principal component analysis", Tunn Undergr sp Tech, 27(1), pp. 133-141 (2012).

37. Kaiser, H.F. "An index of factorial simplicity", Psychometrika, 39(1), pp. 31-36 (1974).

38. Al-Mosawe, A., Kalfat, R., and Al-Mahaidi, R. "Strength of CFRP-steel double strap joints under impact loads using genetic programming", Compos Struct, 160, pp. 1205-1211 (2017).

39. Manouchehrian, A., Sharifzadeh, M., and Moghadam, R.H. "Application of artificial neural networks and multivariate statistics to estimate UCS using textural characteristics", Int. J. Min. Sci. Technol, 22(2), pp. 229-236 (2012).
40. Hamby, D. "A review of techniques for parameter sensitivity analysis of environmental models", Environ Monit Assess, 32(2), pp. 135-154 (1994).

\section{Biographies}

Roshana Sattari received her BSc in Biomedical Engineering from Islamic Azad University of Kerman, Iran, in 2017 and MSc in Materials Science Engineering from Shahid Bahonar University of Kerman, Iran, in 2020 .

Gholam Reza Khayati is currently a faculty member of Materials Science and Engineering at Shahid Bahonar University of Kerman. He received his BSc in Materials Science from Shahid Bahonar University of Kerman, Iran, in 2004; MSc with honors in Materials Science and Engineering from the Department of Materials Science \& Engineering at University of Tehran, Iran; and $\mathrm{PhD}$ in Materials Science from Shiraz University, Iran. He has published more than 40 scientific papers in the field of synthesis and characterization of nanostructure materials. 